\title{
Maladies infectieuses émergentes : prévision et détection
}

\author{
NH Ogden*, P AbdelMalik², JRC Pulliam³
}

\section{Résumé}

Les maladies infectieuses émergentes, y compris le virus du Nil occidental, le syndrome respiratoire aigu sévère (SRAS) et la maladie de Lyme, ont eu un effet direct au Canada, tandis que de nombreuses autres maladies infectieuses émergentes comme le virus Zika, le chikungunya et le virus Ebola menacent les Canadiens qui voyagent. Plus de $75 \%$ des maladies infectieuses émergentes touchant les humains sont, ou étaient à l'origine, des zoonoses (maladies infectieuses transmises d'un animal à un humain). Les maladies infectieuses peuvent émerger de deux principales façons : par des modifications de leurs aires de répartition géographique et par émergence adaptative, un changement génétique chez un microorganisme qui lui permet d'envahir une nouvelle niche, souvent en sautant sur une nouvelle espèce hôte comme l'humain. Les maladies peuvent sembler émerger simplement parce que nous sommes en mesure de les détecter et de les diagnostiquer. La prise en charge des maladies infectieuses émergentes est un rôle essentiel des organismes de santé publique à l'échelle mondiale et constitue un défi considérable sur le plan des soins cliniques. On accorde de plus en plus d'importance à la prévision de l'apparition des maladies infectieuses émergentes afin de "prendre une longueur d'avance ", c'est-à-dire de permettre aux systèmes de santé de mieux se préparer à y réagir, et aux organismes de santé publique, de les prévenir. Les modèles prédictifs servent à estimer le lieu et le moment où des maladies infectieuses émergentes peuvent apparaître ainsi que les niveaux de risque qu'elles posent. L'évaluation des facteurs internes et externes à l'origine d'épisodes d'émergence est de plus en plus utilisée pour prévoir les éclosions de maladies infectieuses émergentes. Actuellement, les changements à l'échelle mondiale conduisent à une hausse des maladies infectieuses émergentes, mais notre capacité à les prévenir et à les gérer s'améliore également. Les méthodes d'analyse et d'exploration basées sur le Web nous permettent de plus en plus de détecter les éclosions de maladies infectieuses émergentes, les techniques modernes de génomique et de bioinformatique augmentent notre capacité à cerner leurs origines génétiques et géographiques, tandis que les progrès réalisés en géomatique et en observation terrestre nous permettent d'effectuer un meilleur suivi des éclosions en temps réel. Toutefois, les maladies infectieuses émergentes resteront un enjeu de santé publique mondiale de premier plan en cette ère de mondialisation où les changements démographiques, climatiques et environnementaux modifient les interactions entre les hôtes et les agents pathogènes de manières qui favorisent la transmission de l'animal vers l'humain et la propagation à l'échelle mondiale.

\author{
Affiliations \\ ${ }^{1}$ Division Science des risques \\ pour la santé publique, \\ Laboratoire national de \\ microbiologie, Agence de la \\ santé publique du Canada, \\ Saint-Hyacinthe (Québec) \\ ${ }^{2}$ Division de la formation et de \\ l'intervention pour le service sur \\ le terrain, Direction générale \\ de l'infrastructure de sécurité \\ sanitaire, Agence de la santé \\ publique du Canada, Ottawa \\ (Ontario) \\ ${ }^{3}$ South African Centre for \\ Epidemiological Modelling \\ and Analysis, Université de \\ Stellenbosch, Cap-Occidental, \\ Afrique du Sud
}

*Correspondance : nicholas. ogden@canada.ca

Citation proposée : Ogden NH, AbdelMalik P, Pulliam JRC. Maladies infectieuses émergentes : prévision et détection. Relevé des maladies transmissibles au Canada. 2017;43(10):232-8. https://doi.org/10.14745/ccdr. v43i10a03f

\section{Introduction}

L'Organisation mondiale de la Santé (OMS) définit une maladie infectieuse émergente (MIE) comme « une maladie qui est apparue au sein d'une population pour la première fois, ou qui peut avoir existé auparavant, mais dont l'incidence ou la portée géographique augmente rapidement » [traduction] (1). Les maladies infectieuses émergentes qui ont directement touché le Canada comprennent le virus du Nil occidental, le syndrome respiratoire aigu sévère (SRAS) et la maladie de Lyme, tandis que de nombreuses autres maladies infectieuses émergentes comme le virus Zika, le chikungunya et le virus Ebola constituent une menace potentielle pour les Canadiens qui voyagent. Une caractéristique commune à de nombreuses maladies infectieuses émergentes est leur capacité à se propager à l'échelle internationale et à affecter la santé mondiale. Par conséquent, la capacité à prévoir l'apparition des maladies infectieuses émergentes, à les détecter et à y réagir est une préoccupation essentielle sur le plan de la santé publique.

Plus de $75 \%$ des maladies infectieuses émergentes touchant les humains (2) sont, ou étaient à l'origine, des zoonoses (maladies infectieuses transmises d'un animal à un humain). Elles sont transmises par différentes voies, y compris les suivantes : contact direct, nourriture, eau potable, eaux de plaisance et vecteurs arthropodes (3). De nombreuses zoonoses (comme le virus du 
Nil occidental et la maladie de Lyme) ne sont transmissibles que d'hôtes réservoirs animaux à l'humain (dans des circonstances normales) sans possibilité de transmission d'humain à humain. Certaines zoonoses sont transmissibles d'humains infectés par des animaux à un autre humain, mais l'efficacité de la transmission d'humain à humain est trop faible pour être viable en l'absence d'hôtes réservoirs animaux (4). Le syndrome respiratoire du Moyen-Orient (SRMO) récemment découvert en est un bon exemple. Il est causé par un coronavirus (CoV-SRMO) transmis par des hôtes réservoirs présumés chez la chauve-souris vers I'humain (6) en passant par des animaux domestiques (chameaux) (5). La transmission de personnes infectées à d'autres personnes par contact étroit (en particulier les travailleurs de la santé) est également possible, mais à ce jour, la transmission d'humain à humain s'est faite par des voies intermittentes qui finissent par s'estomper $(4,7)$. Certaines zoonoses sont hautement transmissibles d'humain à humain; une fois qu'elles sont contractées par un humain, une épidémie peut se répandre au sein de la population, comme ce fut le cas lors de l'éclosion du virus Ebola en Afrique occidentale de 2013 à 2016 (8).

Dans le présent article, nous examinerons brièvement la façon dont les maladies émergent, nous traiterons des facteurs favorisant leur émergence et leur propagation, et nous présenterons les progrès en cours visant à mieux prévoir et détecter les maladies infectieuses émergentes.

\section{Comment les maladies infectieuses émergent-elles?}

Les maladies infectieuses peuvent sembler "émerger " car nous développons la capacité à identifier un nouvel agent pathogène endémique puis à détecter des infections chez l'humain. Récemment, un certain nombre d'agents pathogènes transmis par des tiques, y compris Borrelia mayonii et le virus Heartland, ont été détectés en Amérique du Nord grâce au réexamen méticuleux d'échantillons prélevés chez des patients dont les manifestations cliniques correspondaient à celles d'une maladie infectieuse, mais qui ont obtenu un résultat négatif aux tests de dépistage des agents pathogènes connus $(9,10)$. Toutefois, les maladies infectieuses peuvent émerger de deux principales façons : par des modifications de leurs aires de répartition géographique et par émergence adaptative.

\section{Émergence issue de changements dans l'aire de répartition géographique}

De nombreux épisodes d'émergence de maladies sont associés à des changements de répartition géographique d'agents pathogènes ou de parasites. Cela peut être attribuable à des modifications de l'aire géographique naturelle des animaux vecteurs et hôtes de zoonoses ou encore à la dispersion d'agents pathogènes par des humains infectés, des réservoirs animaux ou des vecteurs. La dispersion peut se faire sur de longues distances, voire à l'échelle mondiale, par des voyageurs infectés, par l'intermédiaire d'activités commerciales (légales et illégales) pouvant impliquer des animaux, des produits d'origine animale ou des vecteurs infectés, et par la migration naturelle d'animaux. Voici des exemples de dispersion de longue distance : introduction du SRAS au Canada par des voyageurs infectés provenant de l'Extrême-Orient (11); l'introduction du virus du Nil occidental en Amérique du Nord par un moustique infecté transporté dans un avion en direction de New York (12); l'introduction du virus Zika sur le continent américain depuis I'Asie en passant par la Micronésie (13). La dispersion peut se faire à l'échelle locale, en raison des déplacements de personnes infectées ou des changements dans la répartition géographique des animaux sauvages hôtes et vecteurs, habituellement dus à des modifications des conditions du milieu qui rendent les nouveaux écosystèmes/environnements propices à la survie d'espèces envahissantes (voir la section ci-dessous intitulée "Prévoir l'émergence des maladies infectieuses »). Voici des exemples de maladies infectieuses émergentes apparues par propagation locale : récente expansion de la maladie de Lyme au Canada en raison de la propagation vers le nord de la tique vectrice Ixodes scapularis provenant des États-Unis (14), et propagation locale du virus Zika en Amérique centrale et du Sud (15).

\section{Émergence adaptative}

L'émergence adaptative correspond à un changement génétique chez un microorganisme entraînant un phénotype capable d'envahir un nouvel écosystème, notamment en sautant sur une nouvelle espèce hôte, y compris l'humain (16). Par exemple, on pense que l'émergence du SRAS et du CoV-SRMO découlerait de changements génétiques améliorant la transmissibilité entre différentes espèces et, peut-être, la pathogénicité $(5,16)$, tandis qu'une mutation du virus chikungunya serait à l'origine de sa transmission par les moustiques-tigres (Aedes albopictus) et de son émergence dans des régions plus tempérées du monde (17). La mutation et le réassortiment génique des virus qui infectent différentes espèces animales sont considérés comme des éléments clés dans l'apparition de nouvelles souches du virus de la grippe pandémique comme pH1N1 (18).

\section{Quelles mesures sont-elles prises en matière de maladies infectieuses émergentes?}

En réaction aux maladies infectieuses émergentes, nous déployons une gamme d'activités allant de la gestion des éclosions à la recherche d'épisodes de maladies infectieuses émergentes en passant par la surveillance des maladies après leur émergence. La recherche vise de plus en plus l'amélioration de notre capacité à prévoir les éclosions de maladies infectieuses émergentes.

\section{Gestion des éclosions}

Les efforts visant à s'assurer de la capacité locale, nationale et internationale à réagir aux éclosions de maladies infectieuses, y compris les maladies infectieuses émergentes, dépassent la portée du présent examen. Cette capacité a sous-tendu la création ou la conception de nombreux organismes de santé publique à l'échelle internationale (p. ex. l'Organisation mondiale de la Santé) et nationale (p. ex. I'Agence de santé publique du Canada) et la mise au point de plans de gestion des maladies infectieuses locales et pandémiques (19). Afin de faciliter davantage la détection, la communication et la gestion des menaces pour la santé, la communauté internationale a convenu 
de respecter le Règlement sanitaire international. Cet instrument juridique contraignant est entré en vigueur en juin 2007 dans le but d'aider la communauté internationale à « prévenir les risques graves pour la santé publique pouvant constituer une menace dans le monde entier, en prenant les mesures qui s'imposent " (20). Son fonctionnement est décrit dans ce qui suit.

\section{Surveillance des épisodes de maladies infectieuses émergentes}

La surveillance des maladies infectieuses émergentes est une activité de santé publique entreprise de façon continue à l'échelle internationale et qui prend de nombreuses formes. D'après l'une des principales capacités requises par le Règlement sanitaire international, tous les États membres de l'Organisation mondiale de la Santé doivent se doter d'une capacité de surveillance des indicateurs et des épisodes. Les États membres sont également tenus de signaler à l'Organisation mondiale de la Santé les événements " inattendus ou inhabituels " (cas humains, fruits et légumes frais contaminés ou vecteurs infectés) en temps opportun (20). Plusieurs types et méthodes de surveillance ont été mis en œuvre au cours des dernières années afin de contribuer à détecter les maladies infectieuses émergentes, et les efforts déployés dans ce domaine se poursuivent. Par exemple, le Système mondial d'alerte rapide (SMAR) est un système d'avertissement en cas de menaces sanitaires et de risques émergents à l'interface humain-animal-écosystème qui reconnaît la nature zoonotique de nombreuses maladies infectieuses émergentes (21). Ce système d'avertissement est géré conjointement par l'Organisation des Nations Unies pour I'alimentation et l'agriculture (ONUAA), I'Office international des épizooties (OIE) et l'Organisation mondiale de la Santé. Un certain nombre de programmes de surveillance " passive » déployés à l'échelle internationale, comme le réseau de surveillance GeoSentinel, un réseau international de cliniques médicales participant sur une base volontaire (22) et de surveillance en laboratoire (23), utilisent des méthodes plus classiques basées sur des indicateurs. L'évolution technologique et la disponibilité des données ont également mené à

l'élaboration de systèmes de surveillance des épisodes, dont un grand nombre décortique le contenu diffusé publiquement sur le Web à la recherche de signaux valides indiquant d'éventuelles menaces émergentes. Citons, par exemple, le programme de surveillance des maladies émergentes (ProMed) (24), HealthMap (25) et MediSys (26). L'un des premiers systèmes du genre est le Réseau mondial d'information en santé publique (RMISP), actuellement entretenu et exploité par l'Agence de la santé publique du Canada. Le RMISP est particulièrement actif en matière de surveillance des maladies infectieuses émergentes, grâce à ses activités continues de développement technique et à son équipe d'analystes multidisciplinaires et multilingues (27).

\section{Prévoir l'émergence des maladies infectieuses}

On accorde de plus en plus d'importance à la prévision de l'apparition des maladies infectieuses émergentes. L'idée est de "prendre une longueur d'avance " afin de permettre aux acteurs de la santé publique de se préparer à réagir aux maladies, voire à les prévenir. L'évaluation des risques exige de se concentrer sur un certain nombre de critères clés associés au degré de menace posée par un agent pathogène ou un épisode, et de tenir compte de la sensibilité des agents pathogènes aux facteurs internes et externes pouvant déclencher une émergence. Les critères clés comprennent la pathogénicité du microorganisme (à savoir, la gravité de la maladie qu'il cause), le potentiel de propagation de l'agent pathogène à l'échelle locale ou internationale, et notre capacité actuelle à le contrôler. Ces différents critères peuvent être synthétisés à l'aide d'une analyse décisionnelle multicritères afin de déterminer quelle mesure prendre ou d'établir des priorités (28). Des études visant à anticiper l'apparition de maladies infectieuses émergentes et à réagir aux maladies infectieuses émergentes existantes sont utilisées pour quantifier ces critères. Citons en exemple l'évaluation de la capacité de propagation des infections à l'échelle internationale (29) et de la possibilité d'une invasion à l'échelle locale (30).

Les évaluations encore plus anticipatives du risque d'émergence de maladies exigent de comprendre les répercussions de facteurs externes. Citons en exemple l'évaluation des effets des changements climatiques et des conditions météorologiques extrêmes comme facteurs réels ou potentiels futurs de l'émergence de maladies sensibles au climat comme la maladie de Lyme au Canada (31) et le virus du Nil occidental aux États-Unis (32). Les autres facteurs externes à l'étude comprennent les niveaux de biodiversité, les changements sur le plan de la biodiversité, de l'agriculture et de l'utilisation des terres (pouvant déclencher une émergence de zoonoses menaçant la population humaine) $(3,33,34)$, et les changements sociaux induits par, notamment, des conflits pouvant favoriser l'émergence ou la réémergence de maladies en nuisant aux programmes de santé publique (35) ou en augmentant la propagation à l'échelle mondiale (36).

Une autre méthode visant à prévoir l'apparition de maladies infectieuses émergentes encore plus en " amont » consiste à identifier les caractéristiques animales ou pathogéniques qui accroissent la possibilité que des microorganismes deviennent des agents pathogènes humains, grâce à leur capacité de transmission d'animaux à l'humain, puis d'humain à humain (37). En général, les virus sont plus susceptibles de devenir des agents pathogènes humains que d'autres microorganismes causant des maladies, comme les microorganismes multi hôtes qui peuvent infecter différentes espèces animales (37-40). Les caractéristiques du génome viral peuvent déterminer le potentiel d'émergence des virus et de leur transmission aux humains (41). Certaines espèces hôtes comme les chauves-souris et les rongeurs ont un potentiel particulièrement élevé de devenir des réservoirs $d$ 'agents pathogènes pouvant être transmis aux humains et conduire à une émergence $(40,42)$.

\section{L'avenir des maladies infectieuses émergentes et de la santé publique}

Les maladies infectieuses émergentes continueront d'être au premier plan des enjeux de santé publique à l'échelle mondiale. Les changements croissants sur le plan du climat, de la biodiversité, de la société et de l'utilisation des terres, dans un monde de plus en plus marqué par les voyages et le commerce, signifient que les risques d'émergence d'agents pathogènes chez les animaux, de transmission aux humains et de propagation rapide à l'échelle mondiale ne cessent d'augmenter. 
Les organismes de santé publique doivent continuer de se concentrer sur trois éléments :

- la capacité à gérer les éclosions de maladies infectieuses émergentes et à s'y préparer;

- la surveillance des épisodes de maladies infectieuses émergentes;

- I'évaluation en amont des risques d'émergence de maladies infectieuses.

Actuellement, les risques de maladies infectieuses émergentes ne cessent d'augmenter, tout comme notre capacité technologique à les gérer. La mise au point de techniques modernes de génomique et de bioinformatique augmente notre capacité à repérer les éclosions de maladies infectieuses émergentes, à localiser leurs sources (43) et à cerner les modifications génétiques pouvant servir à prévoir une émergence (16). Les technologies visant à repérer les épisodes de maladies infectieuses émergentes par l'exploration du Web et l'analyse de signaux dans les médias sociaux sont de plus en plus nombreuses (44). Parallèlement, les avancées dans le domaine de l'observation terrestre (par satellite) améliorent la précision spatio-temporelle des données et la capacité à repérer les changements sur le plan du climat, des conditions météorologiques, des habitats et des caractéristiques socioéconomiques pouvant conduire à l'émergence de maladies (45). Ainsi, on s'attache de plus en plus à surveiller les changements liés aux facteurs déclencheurs de maladies infectieuses émergentes plutôt que les maladies infectieuses émergentes comme telles. II est alors possible de prévenir les maladies infectieuses émergentes.

Il est de plus en plus admis que les maladies infectieuses émergentes sont tout aussi importantes pour la biologie de conservation et la gestion des ressources naturelles que certaines espèces envahissantes (46). Par conséquent, l'approche «Une santé ", selon laquelle la santé humaine, la santé animale et la santé des écosystèmes sont interreliées, améliore l'évaluation des risques, la modélisation prédictive et la détection des maladies infectieuses émergentes (47). En fin de compte, la surveillance des indicateurs et des épisodes s'inscrit dans un continuum de surveillance, fournissant des données différentes, mais précieuses, aux fins d'analyse, d'interprétation et d'intervention. Les efforts visant à relier les différentes approches pour créer des systèmes exhaustifs qui tirent parti des données historiques et contextuelles disponibles permettraient de renforcer davantage la capacité à détecter les maladies infectieuses émergentes, à s'y préparer et à y réagir.

\section{Conclusion}

Nous continuons d'accomplir de grands progrès en matière de prévision, de détection, de prise en charge et de gestion des maladies infectieuses émergentes. Toutefois, les maladies infectieuses émergentes resteront un enjeu de santé publique mondiale de premier plan en cette ère où les changements démographiques, climatiques et environnementaux, y compris la mondialisation, favorisent l'émergence de nouveaux agents pathogènes, leur transmission des animaux aux humains et leur propagation à l'échelle mondiale.

\section{Déclaration des auteurs}

N.H.O., J.R.C.P. - Conceptualisation; N.H.O., P.A. et J.C.R.P., rédaction de l'ébauche originale et examen des ébauches

\section{Conflit d'intérêt}

Aucun.

\section{Références}

1. Organisation mondiale de la Santé. Thèmes de santé : Maladies émergentes. Genève: Organisation mondiale de la Santé. http://www.who.int/topics/emerging_diseases/fr/ [Consulté le 30 août 2017].

2. Woolhouse ME, Gowtage-Sequeria S. Host range and emerging and reemerging pathogens. Emerg Infect Dis. 2005;11:1842-7. DOI (http://dx.doi.org/10.3201/ eid1112.050997). PubMed (https://www.ncbi.nlm.nih. gov/entrez/query.fcgi?cmd=Retrieve\&db=PubMed\&lis t_uids=16485468\&dopt=Abstract).

3. Jones KE, Patel NG, Levy MA, Storeygard A, Balk D, Gittleman JL, Dobson AP, Hudson PJ, Grenfell BT. Global trends in emerging infectious diseases. Nature 2008;451:990-3. DOI (http://dx.doi.org/10.1038/ nature06536). PubMed (https://www.ncbi.nlm.nih.gov/ pubmed/18288193?dopt=Abstract)

4. Lloyd-Smith JO, George D, Pepin KM, Pitzer VE, Pulliam $J R$, et al. Epidemic dynamics at the human-animal interface. Science. 2009;326:1362-7. DOI (http://dx.doi.org/10.1126/ science.1177345). PubMed (https://www.ncbi.nlm.nih. gov/entrez/query.fcgi?cmd=Retrieve \&db=PubMed\&lis t_uids=19965751\&dopt=Abstract).

5. Wang Q, Qi J, Yuan Y, Xuan Y, Han P, Wan Y, Ji W, Li Y, Wu Y, Wang J, Iwamoto A, Woo PC, Yuen KY, Yan J, Lu G, Gao GF. Bat origins of MERS-CoV supported by bat coronavirus HKU4 usage of human receptor CD26. Cell Host Microbe. 2014;16:328-37. DOI (http://dx.doi.org/10.1016/j. chom.2014.08.009). PubMed (https://www.ncbi.nlm.nih. gov/entrez/query.fcgi?cmd=Retrieve\&db=PubMed\&lis t_uids $=25211075 \&$ dopt=Abstract).

6. Haagmans BL, Al Dhahiry SH, Reusken CB, Raj VS, Galiano M, Myers R, Godeke GJ, Jonges M, Farag E, Diab A, Ghobashy H, Alhajri F, Al-Thani M, Al-Marri SA, Al Romaihi $\mathrm{HE}$, Al Khal A, Bermingham A, Osterhaus AD, AlHajri MM, Koopmans MP. Middle East respiratory syndrome coronavirus in dromedary camels: an outbreak investigation. Lancet Infect Dis. 2014;14:140-5. DOI (http://dx.doi.org/10.1016/ S1473-3099(13)70690-X). PubMed (https://www.ncbi.nlm. nih.gov/entrez/query.fcgi?cmd=Retrieve\&db=PubMed\&li st_uids=24355866\&dopt=Abstract).

7. WHO MERS-CoV Research Group. State of knowledge and data gaps of Middle East respiratory syndrome coronavirus (MERS-CoV) in humans. PLoS Curr. 2013;5. PubMed (https:// www.ncbi.nlm.nih.gov/entrez/query.fcgi?cmd=Retrieve \&db= PubMed\&list_uids=24270606\&dopt=Abstract).

8. Coltart CE, Lindsey B, Ghinai I, Johnson AM, Heymann DL. The Ebola outbreak, 2013-2016: old lessons for 
new epidemics. Philos Trans R Soc Lond B Biol Sci. 2017;372: 20160297. DOI (http://dx.doi.org/10.1098/ rstb.2016.0297). PubMed (https://www.ncbi.nlm.nih. gov/entrez/query.fcgi?cmd=Retrieve \&db=PubMed\&lis t_uids $=28396469 \&$ dopt $=$ Abstract) .

9. McMullan LK, Folk SM, Kelly AJ, MacNeil A, Goldsmith CS, Metcalfe MG, Batten BC, Albariño CG, Zaki SR, Rollin PE, Nicholson WL, Nichol ST. A new phlebovirus associated with severe febrile illness in Missouri. N Engl J Med. 2012;367:834-41. DOI (http://dx.doi.org/10.1056/ NEJMoa1203378). PubMed (https://www.ncbi.nlm.nih. gov/entrez/query.fcgi?cmd=Retrieve\&db=PubMed\&lis t_uids=22931317\&dopt=Abstract).

10. Pritt BS, Mead PS, Johnson DK, Neitzel DF, RespicioKingry LB, Davis JP, Schiffman E, Sloan LM, Schriefer ME, Replogle AJ, Paskewitz SM, Ray JA, Bjork J, Steward CR, Deedon A, Lee X, Kingry LC, Miller TK, Feist MA, Theel ES, Patel R, Irish CL, Petersen JM. Identification of a novel pathogenic Borrelia species causing Lyme borreliosis with unusually high spirochaetaemia: a descriptive study. Lancet Infect Dis. 2016;16:556-64. DOI (http://dx.doi.org/10.1016/ S1473-3099(15)00464-8). PubMed (https://www.ncbi.nlm. nih.gov/entrez/query.fcgi?cmd=Retrieve \&db=PubMed\&li st_uids=26856777\&dopt=Abstract).

11. Ma T, Heywood A, Maclntyre CR. Chinese travellers visiting friends and relatives-a review of infectious risks. Travel Med Infect Dis. 2015;13:285-94. DOI (http://dx.doi.org/10.1016/j. tmaid.2015.05.004). PubMed (https://www.ncbi.nlm.nih. gov/entrez/query.fcgi?cmd=Retrieve\&db=PubMed\&lis t_uids=26026478\&dopt=Abstract).

12. Lanciotti RS, Roehrig JT, Deubel V, Smith J, Parker M, Steele K, Crise B, Volpe KE, Crabtree MB, Scherret JH, Hall RA, MacKenzie JS, Cropp CB, Panigrahy B, Ostlund E, Schmitt B, Malkinson M, Banet C, Weissman J, Komar N, Savage HM, Stone W, McNamara T, Gubler DJ. Crise B, Volpe KE, Crabtree MB, Scherret JH, Hall RA, MacKenzie JS, Cropp CB, Panigrahy B, Ostlund E, Schmitt B, Malkinson M, Banet C, Weissman J, Komar N, Savage HM, Stone W, McNamara T, Gubler DJ. Origin of the West Nile virus responsible for an outbreak of encephalitis in the Northeastern United States. Science. 1999;286:2333-7. DOI (http://dx.doi.org/10.1126/ science.286.5448.2333). PubMed (https://www.ncbi.nlm. nih.gov/entrez/query.fcgi?cmd=Retrieve\&db=PubMed\&li st_uids $=10600742 \&$ dopt=Abstract).

13. Baud D, Gubler DJ, Schaub B, Lanteri MC, Musso D. An update on Zika virus infection. Lancet. 2017;S01406736:31450-2. PubMed (https://www.ncbi.nlm.nih.gov/ entrez/query.fcgi?cmd=Retrieve \&db=PubMed\&list_ uids=28647173\&dopt=Abstract).

14. Ogden NH, Radojevic M, Wu X, Duvvuri VR, Leighton, PA Wu J. Estimated effects of projected climate change on the basic reproductive number of the tick vector of Lyme disease Ixodes scapularis. Environ Health Perspect. 2014;122:631-8. PubMed (https://www.ncbi.nlm.nih. gov/entrez/query.fcgi?cmd=Retrieve\&db=PubMed\&lis t_uids $=24627295 \&$ dopt $=$ Abstract)

15. Anderson KB, Thomas SJ, Endy TP. The emergence of Zika virus: A narrative review. Ann Intern Med. 2016;165:175-83. DOI (http://dx.doi.org/10.7326/M16-0617). PubMed (https://
www.ncbi.nlm.nih.gov/entrez/query.fcgi?cmd=Retrieve\&db= PubMed\&list_uids=27135717\&dopt=Abstract).

16. Pepin KM, Lass S, Pulliam JR, Read AF, Lloyd-Smith JO. Identifying genetic markers of adaptation for surveillance of viral host jumps. Nat Rev Microbiol. 2010;8:802-13. DOI (http://dx.doi.org/10.1038/nrmicro2440). PubMed (https:// www.ncbi.nlm.nih.gov/entrez/query.fcgi?cmd=Retrieve\&db= PubMed\&list_uids=20938453\&dopt=Abstract).

17. Tsetsarkin KA, Vanlandingham DL, McGee CE, Higgs $\mathrm{S}$. A single mutation in chikungunya virus affects vector specificity and epidemic potential. PLoS Pathog. 2007;3(12):e201. DOI (http://dx.doi.org/10.1371/journal. ppat.0030201). PubMed (https://www.ncbi.nlm.nih.gov/ entrez/query.fcgi?cmd=Retrieve\&db=PubMed\&list_ uids=18069894\&dopt=Abstract).

18. Guan Y, Vijaykrishna D, Bahl J, Zhu H, Wang J, Smith GJ. The emergence of pandemic influenza viruses. Protein Cell. 2010;1:9-13. DOl (http://dx.doi.org/10.1007/ s13238-010-0008-z). PubMed (https://www.ncbi.nlm.nih. gov/entrez/query.fcgi?cmd=Retrieve\&db=PubMed\&lis t_uids=21203993\&dopt=Abstract).

19. Organisation mondiale de la Santé. Préparation et riposte aux situations d'urgence. Genève : Organisation mondiale de la Santé. http://www.who.int/csr/fr/ [Consulté le 30 août 2017].

20. Organisation mondiale de la Santé. Thèmes de santé; Règlement sanitaire international (RSI). Genève: Organisation mondiale de la Santé. http://www.who.int/topics/ international_health_regulations/fr/ [Consulté le 30 août 2017].

21. GLEWS+: Objectives. Rome: Food and Agriculture Organization. Joint publication with the World Organization for Animal Health (OIE) and the World Health Organization. http://www.glews.net/?page_id=34 [Consulté le 30 août 2017].

22. Torresi J, Leder K. Defining infections in international travellers through the GeoSentinel surveillance network. Nat Rev Microbiol. 2009;7(12):895-901. PubMed (https://www. ncbi.nlm.nih.gov/entrez/query.fcgi?cmd=Retrieve \&db=PubM ed\&list_uids=19881521\&dopt=Abstract).

23. CDC. Emerging Infections Programs: About the Emerging Infections Programs. Atlanta: CDC Division of Preparedness and Emerging Infections. https://www.cdc.gov/ncezid/dpei/ eip/index.html [Consulté le 30 août 2017].

24. ProMed. About ProMED-mail. Brookline (MA): International Society for Infectious Diseases. https://www.promedmail.org/ aboutus/ [Consulté le 30 août 2017].

25. Computational Epidemiology Group. HealthMap: [ ] alerts for Alerts from past week. Boston (MA): Boston Children's Hospital. http://www.healthmap.org/ [Consulté le 30 août 2017].

26. DG Joint Research Centre. MediSys: Most active topics. European Commission. http://medisys.newsbrief.eu/medisys/ homeedition/en/home.html [Consulté le 30 août 2017].

27. Dion M, AbdelMalik P, Mawudeku A. Les données massives et le Réseau mondiale d'information en santé publique (RMISP). Relevé de maladies transmissibles au Canada. 
2015;41-9:248-50. https://www.canada.ca/content/dam/ phac-aspc/migration/phac-aspc/publicat/ccdr-rmtc/15vol41/ dr-rm41-09/assets/pdf/15vol41_09-fra.pdf

28. Hongoh V, Michel P, Gosselin P, Samoura K, Ravel A, Campagna C, Cissé HD, Waaub JP. Multi-stakeholder decision aid for improved prioritization of the public health impact of climate sensitive infectious diseases. Int J Environ Res Public Health. 2016;13:419. DOI (http://dx.doi. org/10.3390/ijerph13040419). PubMed (https://www.ncbi. nlm.nih.gov/entrez/query.fcgi?cmd=Retrieve\&db=PubMed\&l ist_uids=27077875\&dopt=Abstract).

29. Johansson MA, Powers AM, Pesik N, Cohen NJ, Staples JE. Nowcasting the spread of Chikungunya virus in the Americas. PLoS One. 2014;9:e104915. DOI (http://dx.doi.org/10.1371/ journal.pone.0104915). PubMed (https://www.ncbi.nlm. nih.gov/entrez/query.fcgi?cmd=Retrieve\&db=PubMed\&li st_uids $=25111394 \&$ dopt $=$ Abstract $)$.

30. Ng V, Fazil A, Gachon P, Deuymes G, Radojević M, Mascarenhas M, Garasia S, Johansson MA, Ogden $\mathrm{NH}$. Assessment of the probability of autochthonous transmission of Chikungunya virus in Canada under recent and projected climate change. Environ Health Perspect. 2017;125(6):067001. DOI (http://dx.doi.org/10.1289/ EHP669). PubMed (https://www.ncbi.nlm.nih.gov/ entrez/query.fcgi?cmd=Retrieve\&db=PubMed\&list_ uids=28731409\&dopt=Abstract).

31. Ebi KL, Ogden NH, Semenza JC, Woodward A (2017) Detecting and attributing health burdens to climate change. Environ Health Perspect. 2017;125(8):085004. DOI (http:// dx.doi.org/10.1289/EHP1509). PubMed (https://www.ncbi. $\mathrm{nlm}$.nih.gov/entrez/query.fcgi?cmd=Retrieve\&db=PubMed\&l ist_uids=28796635\&dopt=Abstract).

32. Chung WM, Buseman CM, Joyner SN, Hughes SM, Fomby TB, Luby JP, Haley RW. The 2012 West Nile encephalitis epidemic in Dallas, Texas. JAMA. 2013;310:297-307. DOI (http://dx.doi.org/10.1001/jama.2013.8267). PubMed (https://www.ncbi.nlm.nih.gov/entrez/query.fcgi?cmd=Retrie ve\&db=PubMed\&list_uids=23860988\&dopt=Abstract)

33. Pulliam JR, Epstein JH, Dushoff J, Rahman SA, Bunning $M$, Jamaluddin AA, Hyatt AD, Field HE, Dobson AP, Daszak P; Henipavirus Ecology Research Group (HERG). Agricultural intensification, priming for persistence and the emergence of Nipah virus: a lethal bat-borne zoonosis. J R Soc Interface. 2012;9:89-101. DOI (http://dx.doi. org/10.1098/rsif.2011.0223). PubMed (https://www.ncbi.nlm. nih.gov/entrez/query.fcgi?cmd=Retrieve\&db=PubMed\&li st_uids=21632614\&dopt=Abstract).

34. McFarlane RA, Sleigh AC, McMichael AJ. Land-use change and emerging infectious disease on an island continent. Int J Environ Res Public Health. 2013;10:2699-719. DOI (http:// dx.doi.org/10.3390/ijerph10072699). PubMed (https://www. ncbi.nlm.nih.gov/entrez/query.fcgi?cmd=Retrieve\&db=PubM ed\&list_uids=23812027\&dopt=Abstract).

35. Semenza JC, Lindgren E, Balkanyi L, Espinosa L, Almqvist MS, Penttinen P, Rocklöv J. Determinants and Drivers of Infectious Disease Threat Events in Europe. Emerg Infect Dis. 2016;22:581-9. DOI (http://dx.doi.org/10.3201/ eid2204.151073). PubMed (https://www.ncbi.nlm.nih.
gov/entrez/query.fcgi?cmd=Retrieve $\& \mathrm{db}=$ PubMed\&lis t_uids=26982104\&dopt=Abstract).

36. Ciervo A, Mancini F, di Bernardo F, Giammanco A, Vitale G, Dones P, Fasciana T, Quartaro P, Mazzola G, Rezza G. Louseborne relapsing fever in young migrants, Sicily, Italy, July-September 2015. Emerg Infect Dis. 2016;22:152-3. DOI (http://dx.doi.org/10.3201/eid2201.151580). PubMed (https://www.ncbi.nlm.nih.gov/entrez/query.fcgi?cmd=Retrie ve\&db=PubMed\&list_uids=26690334\&dopt=Abstract).

37. Plowright RK, Parrish CR, McCallum H, Hudson PJ, Ko Al, Graham AL, Lloyd-Smith JO. Pathways to zoonotic spillover. Nat. Rev. Microbiol. 2017;15(8):502-10. DOl (http://dx.doi. org/10.1038/nrmicro.2017.45). PubMed (https://www.ncbi. $\mathrm{nlm}$.nih.gov/entrez/query.fcgi?cmd=Retrieve \&db=PubMed\&l ist_uids=28555073\&dopt=Abstract).

38. Pulliam JRC. Viral host jumps: moving toward a predictive framework. EcoHealth. 2008;5:80-91. DOI (http://dx.doi. org/10.1007/s10393-007-0149-6). PubMed (https://www. ncbi.nlm.nih.gov/entrez/query.fcgi?cmd=Retrieve\&db=PubM ed\&list_uids=18648800\&dopt=Abstract).

39. Woolhouse ME, Brierley L, McCaffery C, Lycett S. Assessing the epidemic potential of RNA and DNA viruses. Emerg Infect Dis. 2016;22:2037-44. DOI (http://dx.doi.org/10.3201/ eid2212.160123). PubMed (https://www.ncbi.nlm.nih. gov/entrez/query.fcgi?cmd=Retrieve\&db=PubMed\&lis t_uids=27869592\&dopt=Abstract)

40. Olival, KJ, Hosseini PR, Zambrana-Torrelio C, Ross N, Bogich TL, Daszak P. Host and viral traits predict zoonotic spillover from mammals. Nature 2017;546:646-50. DOl (http://dx.doi. org/10.1038/nature22975). PubMed (https://www.ncbi.nlm. nih.gov/entrez/query.fcgi?cmd=Retrieve \&db=PubMed\&li st_uids=28636590\&dopt=Abstract).

41. Geoghegan JL, Senior AM, Di Giallonardo F, Holmes EC. Virological factors that increase the transmissibility of emerging human viruses. Proc Natl Acad Sci U S A. 2016;113:4170-5. DOI (http://dx.doi.org/10.1073/ pnas.1521582113). PubMed (https://www.ncbi.nlm.nih. gov/entrez/query.fcgi?cmd=Retrieve\&db=PubMed\&lis t_uids=27001840\&dopt=Abstract).

42. Han BA, Schmidt JP, Bowden SE, Drake JM. Rodent reservoirs of future zoonotic diseases. Proc Natl Acad Sci U S A 2015;112:7039-44. DOI (http://dx.doi.org/10.1073/ pnas.1501598112). PubMed (https://www.ncbi.nlm.nih. gov/entrez/query.fcgi?cmd=Retrieve \&db=PubMed\&lis t_uids=26038558\&dopt=Abstract).

43. Gilmour MW, Graham M, Reimer A, Van Domselaar G. Public health genomics and the new molecular epidemiology of bacterial pathogens. Public Health Genomics 2013;16:25-30. DOI (http://dx.doi. org/10.1159/000342709). PubMed (https://www.ncbi.nlm. nih.gov/entrez/query.fcgi?cmd=Retrieve \&db=PubMed\&li st_uids=23548714\&dopt=Abstract).

44. Quade P, Nsoesie EO. A platform for crowdsourced foodborne illness surveillance: description of users and reports. JMIR Public Health Surveill. 2017;3(3):e42. DOI (http://dx.doi.org/10.2196/ publichealth.7076). PubMed (https://www.ncbi.nlm.nih. gov/entrez/query.fcgi?cmd=Retrieve\&db=PubMed\&lis t_uids=28679492\&dopt=Abstract). 
45. Brazeau S, Aubé G, Turgeon P, Kotchi S-O, Michel P. Tele-epidemiology: advancing the application of earth observation to public health issues in Canada. New York: Earthzine; 2014. https://earthzine.org/2014/05/02/ tele-epidemiology-advancing-the-application-of-earthobservation-to-public-health-issues-in-canada/

46. Dunn AM, Hatcher MJ. Parasites and biological invasions: parallels, interactions, and control. Trends Parasitol. 2015;31:189-99. DOI (http://dx.doi.org/10.1016/j. pt.2014.12.003). PubMed (https://www.ncbi.nlm.nih. gov/entrez/query.fcgi?cmd=Retrieve $\& d b=$ PubMed\&lis t_uids $=25613560 \& d o p t=$ Abstract).

47. Woolhouse M. How to make predictions about future infectious disease risks. Philos Trans R Soc Lond B Biol Sci. 2011;366:2045-54. DOI (http://dx.doi.org/10.1098/ rstb.2010.0387). PubMed (https://www.ncbi.nlm.nih. gov/entrez/query.fcgi?cmd=Retrieve\&db=PubMed\&lis t_uids=21624924\&dopt=Abstract).

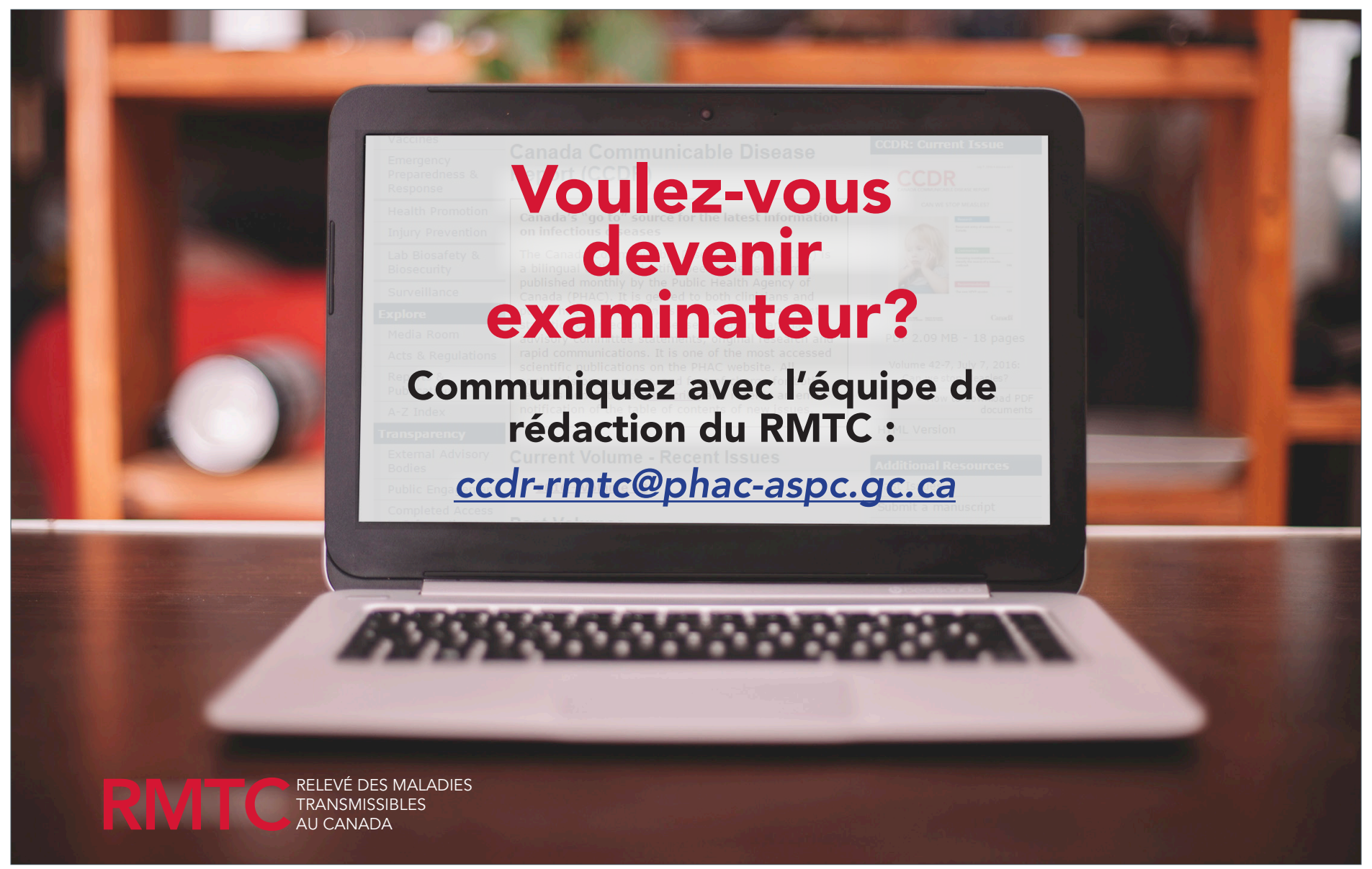

